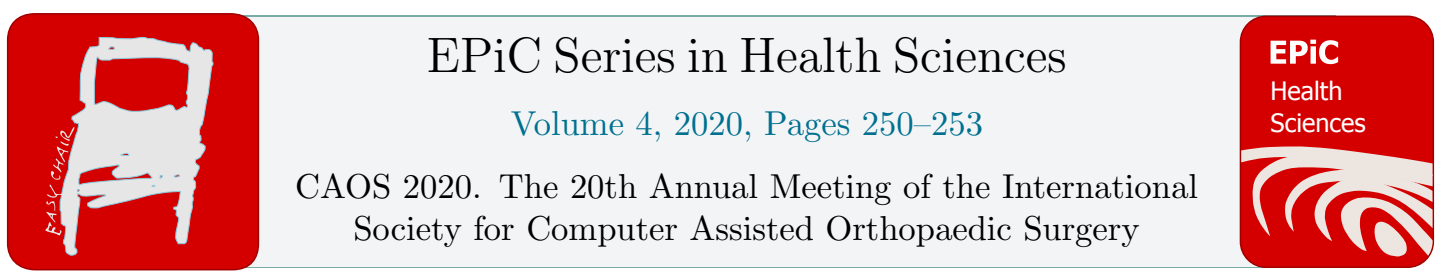

\title{
A Novel Computer-Assisted Workflow for Treatment of Osteochondral Lesions in the Knee
}

\author{
Fabio Tatti ${ }^{1}$, Hisham Iqbal ${ }^{1}$, Branislav Jaramaz ${ }^{2}$ and \\ Ferdinando Rodriguez y Baena ${ }^{1}$ \\ ${ }^{1}$ Department of Mechanical Engineering, Imperial College London, London, UK \\ ${ }^{2}$ Smith \& Nephew Inc., Pittsburgh, PA, U.S.A. \\ \{f.tatti, hisham.iqbal13, f.rodriguez\}@imperial.ac.uk \\ branko.jaramaz@smith-nephew.com
}

\section{Introduction}

Despite a slow uptake, there have been considerable technological developments in ComputerAssisted Orthopaedic Surgery (CAOS), and these systems are now becoming more prevalent, especially in knee arthroplasty (Picard et al. 2019). CAOS systems have the potential to improve the accuracy and repeatability of surgical procedures by means of digital preoperative planning and intraoperative tracking of the patient and surgical instruments. Besides improving the outcome of classical orthopaedic procedures, these systems also allow the design of new types of surgical interventions, based on intraoperative quantitative data and tailored to the needs of each patient.

One area where the accuracy and repeatability of computer-assisted interventions could prove especially beneficial is the treatment of osteochondral defects (OCD). OCDs represent a common problem in the patient population, and are often a cause of pain and discomfort (Solheim et al. 2016). The use of synthetic implants is a valid option for patients who cannot be treated with regenerative methods (Bollars et al. 2012; Fuchs et al. 2018), but the outcome can be negatively impacted by incorrect positioning of the implant and lack of congruency with the surrounding anatomy (Becher et al. 2008; Martinez-Carranza et al. 2013).

In this paper, we present a novel computer-assisted surgical workflow for the treatment of osteochondral defects. The software we developed automatically selects the implant that most closely matches the patient's anatomy and computes the best pose. By combining this software with the existing capabilities of the Navio ${ }^{\mathrm{TM}}$ surgical system (Smith \& Nephew inc.), we were able to create a complete workflow that incorporates both surgical planning and assisted bone preparation. 


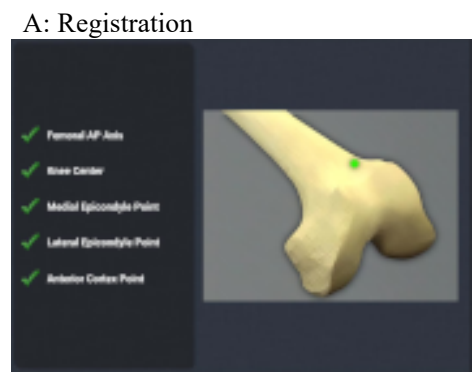

D: Implant selection

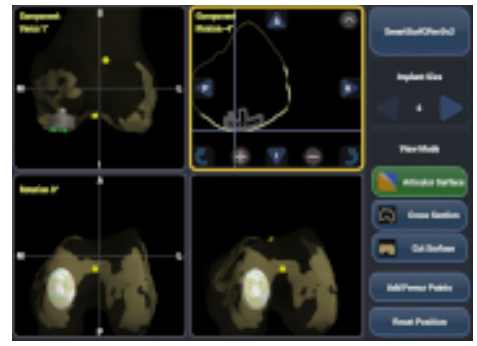

B: Bone modelling

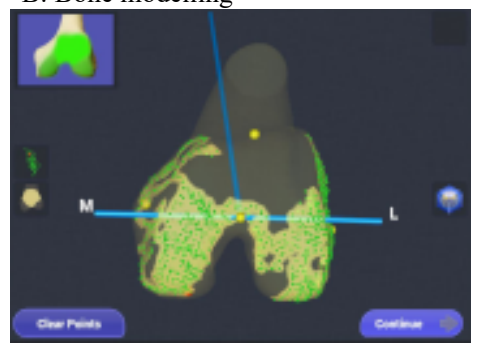

E: Bone preparation

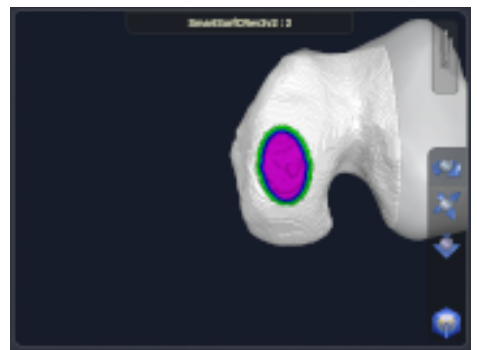

C: Defect mapping

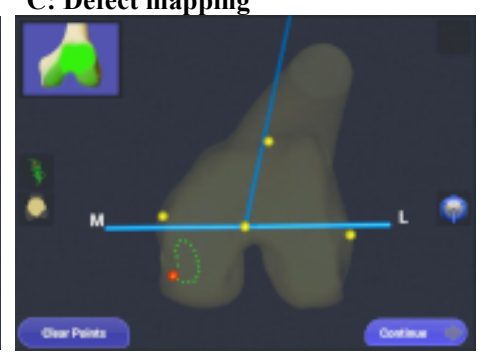

F: Implant placement

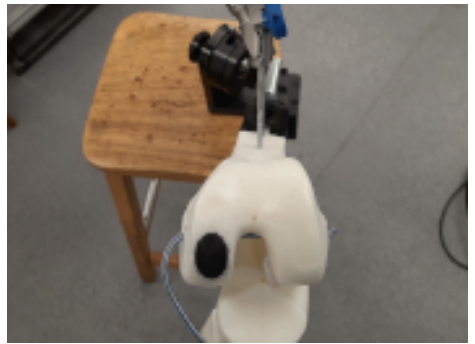

Figure 1: Steps of the workflow. Steps based on the new software (C,D) are highlighted in bold, while the remaining steps $(\mathrm{A}, \mathrm{B}, \mathrm{E})$ are based on the Navio ${ }^{\mathrm{TM}} \mathrm{PFA}$ workflow.

\section{Materials and Methods}

\subsection{Implants}

For this work we used a custom set of implant geometries for the treatment of knee osteochondral defects. These were developed by collecting a large dataset of defect geometries extracted from medical scans and modelling their shape through Principal Component Analysis. The development of the implants was presented and discussed at a previous CAOS meeting (Tatti et al. 2019).

The choice to use this set of implants does not restrict the applicability of the method. Indeed, the workflow can be generalised to work with any family of implants.

\subsection{Defect Modelling and Implant Selection}

The new surgical workflow is built around a software module that takes as input a 3D model of the patient's femur and a vector of points representative of the defect contour. The femur 3D model is assumed to be constructed by means of a statistical shape model (Sarkalkan et al. 2014), therefore ignoring the presence of a defect and reconstructing the "missing" cartilage surface, as if the defect were not present.

The defect's size and shape were characterized by first projecting the OCD contour on a 2D plane and computing the smallest ellipse that contained all points, then cropping out the femur surface falling within the ellipse and parametrizing its shape through the following model, which describes a paraboloid with a free rotation around the $\mathrm{z}$ axis : 
(Eq. 1) $\left\{\begin{array}{c}x^{\prime}=\left(x-x_{0}\right) \cos \theta+\left(y-y_{0}\right) \sin \theta \\ y^{\prime}=-\left(x-x_{0}\right) \sin \theta+\left(y-y_{0}\right) \cos \theta \\ z=a x^{\prime 2}+b y^{\prime 2}+z_{0}\end{array}\right.$

$\theta=0$ corresponds to $x^{\prime}$ being aligned with the major axis of the contour ellipse. Model parameters are identified using numerical non-linear optimization.

Each of the implants in the library that we used in this work can be described with the same set of parameters from Eq.1. We can therefore select the best implant for a given defect by comparing the defect's parametric description with that of the implants. The implant's best pose is then computed using the ICP algorithm (Besl and McKay 1992) to co-register the implant's and femur's outer surfaces.

\section{Results}

After implementing the defect parameterisation and implant selection routines in $\mathrm{C}++$, we combined these tools with the existing capabilities of the $\mathrm{Navio}^{\mathrm{TM}}$ surgical system, to create a complete surgical workflow. The stages of the complete workflow are shown in Fig. 1. As per the existing surgical registration procedure for the robot, the surgeon is first asked to collect the position of various anatomical landmarks, to register the position of the patient, and then they paint along the exposed surface of the femur using the system's tracked probe, to model the bone anatomy. After this stage, the surgeon traces the outer edge of the defect and the collected information is then used to select the optimal implant and its pose. Finally, the system generates a cut-plan for the bone in order to correctly place the implant and the surgeon executes it using the system's burring tool.

We conducted preliminary testing by simulating the presence of an osteochondral defect on plastic femur models and using the new workflow to model the defects, select an appropriate implant and pose, and prepare the bone for the implant's insertion. The testing was successful and demonstrated that the workflow can be used to select and position an appropriate implant for a given defect. Fig. 1,E shows the successful placement of an implant prototype.

\section{Discussion}

This work presents a proof-of-concept demonstration of a computer-assisted surgical workflow for the treatment of osteochondral defects in the knee joint. We integrated our software within a commercial surgical system and demonstrated the usability of the complete workflow.

The use of CAOS systems has been shown to improve the accuracy and repeatability of implant placement in classical procedures such as total and unicondylar knee arthroplasties, e.g. (Chauhan et al. 2004; Anderson et al. 2005). Our goal was to bring these benefits to osteochondral defect repair, where implant mispositioning and lack of anatomical congruency can also negatively impact success (Becher et al. 2008; Martinez-Carranza et al. 2013).

The system we present can improve the surgical outcome in two aspects: firstly, it selects an implant and its ideal pose based on a quantitative measurement of similarity and congruency with the patient's anatomy; secondly, it assists the surgeon during bone removal, increasing the accuracy of the implant's placement.

The results currently available are preliminary, and more extensive testing on plastic models, as well as a cadaveric trial, are planned for future studies. 


\section{Acknowledgements}

The authors wish to thank TJ Smith \& Nephew Limited for technical support and insights into the Navio $^{\text {TM }}$ system. This study was funded by INNOVATE UK under project number 103950.

\section{References}

Anderson KC, Buehler KC, Markel DC (2005) Computer assisted navigation in total knee arthroplasty: Comparison with conventional methods. J Arthroplasty 20:132-138. https://doi.org/10.1016/j.arth.2004.10.004

Becher C, Huber R, Thermann H, et al (2008) Effects of a contoured articular prosthetic device on tibiofemoral peak contact pressure: A biomechanical study. Knee Surgery, Sport Traumatol Arthrosc 16:56-63. https://doi.org/10.1007/s00167-007-0416-7

Besl P, McKay N (1992) A Method for Registration of 3-D Shapes. IEEE Trans. Pattern Anal. Mach. Intell. 14:239-256

Bollars P, Bosquet M, Vandekerckhove B, et al (2012) Prosthetic inlay resurfacing for the treatment of focal, full thickness cartilage defects of the femoral condyle: A bridge between biologics and conventional arthroplasty. Knee Surgery, Sport Traumatol Arthrosc 20:1753-1759. https://doi.org/10.1007/s00167-011-1757-9

Chauhan SK, Scott RG, Breidahl W, Beaver RJ (2004) Computer-assisted knee arthroplasty versus a conventional jig-based technique. A randomised, prospective trial. J Bone Jt Surg - Ser B 86:372377. https://doi.org/10.1302/0301-620X.86B3.14643

Fuchs A, Eberbach H, Izadpanah K, et al (2018) Focal metallic inlay resurfacing prosthesis for the treatment of localized cartilage defects of the femoral condyles: a systematic review of clinical studies. Knee Surgery, Sport Traumatol Arthrosc 26:2722-2732. https://doi.org/10.1007/s00167017-4714-4

Martinez-Carranza N, Berg HE, Hultenby K, et al (2013) Focal knee resurfacing and effects of surgical precision on opposing cartilage. A pilot study on 12 sheep. Osteoarthr Cartil 21:739-745. https://doi.org/10.1016/j.joca.2013.02.004

Picard F, Deakin AH, Riches PE, et al (2019) Computer assisted orthopaedic surgery: Past, present and future. Med Eng Phys 72:55-65. https://doi.org/10.1016/j.medengphy.2019.08.005

Sarkalkan N, Weinans H, Zadpoor AA (2014) Statistical shape and appearance models of bones. Bone 60:129-140. https://doi.org/10.1016/j.bone.2013.12.006

Solheim E, Krokeide AM, Melteig P, et al (2016) Symptoms and function in patients with articular cartilage lesions in 1,000 knee arthroscopies. Knee Surgery, Sport Traumatol Arthrosc 24:1610 1616. https://doi.org/10.1007/s00167-014-3472-9

Tatti F, Farley D, Jaramaz B, Rodriguez y Baena F (2019) Data - Driven Design of an Implant Library for Osteochondral Lesion Repair in the Knee Joint. In: CAOS 2019. New York 